\title{
Off-Peak: An Examination of Ordinary Player Experience
}

\author{
April Tyack, Elisa D. Mekler \\ firstname.lastname@aalto.fi \\ Aalto University \\ Espoo, Finland
}

\begin{abstract}
Videogames' increasing cultural relevance and suffusion into everyday use contexts suggests they can no longer be considered novelties. Broadly speaking, games research at $\mathrm{CHI}$ has concerned two forms of peak experience-historically, research aimed to support flow, or maximise enjoyment and positive emotions; more recently, scholarship engages with more varied experiences of intense emotion, such as emotional challenge. In different ways, both approaches emphasise extra-ordinary player experience (PX). Conversely, videogame play and $\mathrm{PX}$ have become more routine-indeed, more ordinary-as the medium's cultural presence grows. In this paper, we argue that HCI games research is conceptually ill-equipped to investigate these increasingly common and often desirable experiences. We conceptualise "ordinary player experience" - as familiar, emotionally moderate, co-attentive, and abstractly memorable - articulating a phenomenon whose apparent mundanity has seen it elude description to date. We discuss opportunities to productively employ ordinary PX in HCI games research, alongside conceptual implications for PX and player wellbeing.
\end{abstract}

\section{CCS CONCEPTS}

- Human-centered computing $\rightarrow \mathrm{HCI}$ theory, concepts and models; • Applied computing $\rightarrow$ Computer games.

\section{KEYWORDS}

player experience, ordinary experience, videogames, peak experience, optimal experience

\section{ACM Reference Format:}

April Tyack, Elisa D. Mekler. 2021. Off-Peak: An Examination of Ordinary Player Experience. In CHI Conference on Human Factors in Computing Systems (CHI '21), May 8-13, 2021, Yokohama, Japan. ACM, New York, NY, USA, 12 pages. https://doi.org/10.1145/3411764.3445230

\section{INTRODUCTION}

Games and play research at $\mathrm{CHI}$ has increasingly recognised the broad variation in player experience (PX): in recent years, experiences such as meaning [61], appreciation [16], and emotional challenge [17] have gained traction alongside more foundational experiences of enjoyment [94], flow [144], and positive affect [90]. These constructs reflect varying aims and interests that have emerged and developed within the broader domain of HCI games research.

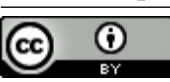

This work is licensed under a Creative Commons Attribution International 4.0 License.

CHI '21, May 8-13, 2021, Yokohama, Japan

(C) 2021 Copyright held by the owner/author(s)

ACM ISBN 978-1-4503-8096-6/21/05.

https://doi.org/10.1145/3411764.3445230
Each strand of PX scholarship is alike, however, in locating videogames' academic significance in the extra-ordinary experiences they support. This perspective is most overt in scholars' exhortations of the medium [e.g., "the uniqueness of gaming experience is one important reason for the great success of digital games in general"; 158, p. 244], but is inherent to all research where games are used to improve another activity ${ }^{1}$.

Videogames have also attained mainstream success: industry reports estimate that roughly two-thirds of people in Western countries play videogames $[19,20,44,83]$; in Australia and New Zealand (for which detailed statistics are available), people play for over 80 minutes a day on average [19, 20]. Videogames, and the experiences they evoke, are rarely exceptional or novel for these people: play has become "a healthy part of [the] everyday routine" [19, p. 61]. Seen in this way, the unique, intense, or memorable experiences that structure much of HCI games research are highly atypical [112] - indeed, the experiences that videogames reliably and desirably evoke are more often ordinary. The ways that experience is conceptualised in HCI games scholarship is yet to account for the appeal of ordinary PX.

In this paper, we argue for the importance of ordinary PX, synthesising developments in HCI, consumer research, and game studies to identify its primary elements. In the following, we conceptualise ordinary PX in terms of its salient properties - as being familiar, emotionally moderate, co-attentive, and abstractly memorable - before outlining its applications to $\mathrm{HCI}$ games research. The present work therefore contributes a vocabulary for PX scholars to discuss and study a prevalent yet overlooked phenomenon. In making ordinary PX available for further theoretical and empirical inquiry, we articulate conceptual implications with respect to other topics of interest within HCI games research, such as wellbeing. In complicating the centrality of extra-ordinary experiences in conceptual and practice-led HCI games scholarship, our conceptualisation extends PX theory development and contributes to the ongoing maturation of the field [104]. Moreover, our work demonstrates that established PX constructs and methods are poorly-suited to account for arguably the most common player experiences: players are not exclusively drawn to play videogames to experience maximal interest, curiosity, need satisfaction, or strong emotions - constructs that have to date consumed the bulk of attention in HCI games research. Finally, conceptualising ordinary PX contributes to a more comprehensive view of extra-ordinary player experiences, and a shared vocabulary that helps articulate its fundamental qualities.

\footnotetext{
${ }^{1}$ Carter et al. [23] alternatively interpret this research as devaluing games as objects of study, but we believe it more likely reflects an optimism towards the medium's transformative potential.
} 


\section{EXTRA-ORDINARY EXPERIENCE}

The study of extra-ordinary experience - its varieties, characteristics, and antecedents - has a long tradition in psychology and other fields concerned with human experience. Maslow, for instance, described peak experiences as rare and unexpected "moments of highest happiness and fulfillment" [92, p. 69] that surpass other experiences in richness, intensity, and meaningfulness. Peak experiences are usually associated with religious or mystical epiphanies that transcend the self [120]. Peak performance, in contrast, is understood as "an episode of superior functioning" [120, p. 1361] and "behavior which exceeds typical behavior" [120, p. 1362] in efficiency, creativity, or productivity. Finally, while flow has much in common with peak experience and performance, it refers specifically to a state of optimal experience. Flow states are characterised by the perception of activities as highly challenging, yet manageable [i.e., challenge-skill balance; 33], involving high concentration and enjoyment.

Consumer research has likewise sought to capture and facilitate extra-ordinary experiences [57], initially conceptualised as emotionally intense, positive, and intrinsically enjoyable, with a "sense of newness of perception and process" [9, p. 41]. Duerden et al. have since derived a more granular taxonomy of extra-ordinary experiences [43, 131]. In their framework, memorable experiences "hold an individual's attention and produce ... strong emotions" [43, p. 204], whereas meaningful experiences also involve the "discovery of significant and personally relevant insights" [43, p. 206]. Finally, transformational experiences are understood as intensely emotional and memorable experiences that prompt meaningful revelations and enduring change in personal beliefs, self-perception, and behaviour.

In the major tradition of games research, the study of games and play is bound up with extra-ordinary experience from the beginning [e.g., see 107]. An early study of game enjoyment took direction from flow theory, claiming that "satisfaction, enjoyment, fun, and other aspects of ... experience have been widely regarded as the essence of play" [56, p. 729]. A similarly essentialising account has obtained in more recent enjoyment research, whereby videogames are considered to "offer some of the most intense, rich and engaging experiences of all interactive products" [94, p. 927].

This apparently defining quality is taken for granted again, most notably, in GameFlow [144], whose turn to (player) experience was predicated on the limits of videogame research applying isolated concepts from media theory, such as genre and transportation. In taking optimal experience as its foundation, however, GameFlow itself arguably also comes to represent a narrow view of PX. A similar perspective prevails in more recent work, including research investigating peak-end effects (with respect to challenge-skill balance) in videogame play:

"Games provide an interesting platform for examining peak-end effects because the activities involved are designed to be highly engaging and immersive ... games may also generate stronger momentary experiences than other forms of daily interactive tasks, allowing more sensitive experimental examination of peak-end effects" [52, p. 5608].
Similar claims are made in self-determination theory (SDT) literature addressing game overuse [e.g., 132]. In particular, the need density hypothesis is predicated on the notion that "few activities ... have the capacity to provide need satisfaction with high degrees of [immediacy, consistency, or density] - let alone all of them-the way that video games do" [125, p. 102]. Indeed, videogame play is said to differ from "many other leisure activities, [as] there are no limits to the imagination and resources that game developers will dedicate to creating [psychologically satisfying] in-game experiences" [132, p. 523], which are apparently comparable to recreational drug use: "just as cocaine directly and immediately gives addicts that [dopamine] rush, so too [does] the immediacy and density of intrinsic need satisfaction in [massively multiplayer online games]" [125, p. 109]. SDT-based HCI games research does not appear to frequently engage with the need density hypothesis [149]; however, it remains likely that SDT's characterisation of videogames as inherently facilitating extra-ordinary PX has been influential.

Arguing for the merits of studying both positive and negative emotional responses to play, Birk et al. were among the earliest within HCI to critique the focus on enjoyment, flow, and immersion in PX research [14]. Operating on similar terms, the nascent HCI games research on mixed affect [16], serious experience [58, 91], and reflection [77, 95] has aimed to extend PX research beyond fun.

However, much of this literature also works from the basis that games evoke extra-ordinary experiences. Bopp et al. argue that "emotionally moving game experiences ... sometimes even had a deep personal impact on players" [16, p. 3003], and "often 'linger' with players for a long time" [16, p. 3005], suggesting both strong emotion and memorability. Finally, emotionally challenging PX is also predicated on videogames' capacity to elicit "intense negative emotions" [17, p. 8] that "offer more diverse and unique gaming experiences" [113, p. 10], purportedly supporting the extra-ordinary experience of reflection.

Many PX studies are not explicitly based in paradigms of extraordinary experience [23]; for example, works that employ selfdetermination theory [see 149 , for a review]. Constructs such as need satisfaction or enjoyment do not themselves connote extraordinary PX - however, an implicit methodological bias manifests in quantitative work that regards higher scale ratings as inherently constituting a 'better' experience.

In a similar way, the HCI games literature does contain more measured claims regarding extra-ordinary PX. Recent work has questioned the focus on "relatively long, highly interactive and even intense [play] sessions" [34, p. 133] in PX research, pointing to the ways that Neko Atsume [54] supports habitual play marked by frequent disengagement. Interrogating cultural distinctions between 'casual' and 'hardcore' games, Kultima foregrounds the rigidity of what is considered desirable PX, observing that "we have already managed to build some pivotal premises for digital game design ... for example, the notions of challenge, immersion, flow and meaningful actions ... Why do games need to be highly challenging? Why do I need to devote my entire attention to the game and become immersed in its world? Why do I need to feel the flow of the game experience? ... What is really a good game?" [85]. Finally, while a study of player emotions posits that "a good game is likely to elicit a strong overall emotional response" [123, p. 346], the authors also 
acknowledge that "games are not always played in order to feel strong emotions” [123, p. 346].

Extra-ordinary experiences have played a vital role within HCI games scholarship to date. Despite being somewhat rare experiences, the prominence of extra-ordinary PX remains largely unquestioned. Indeed, similar trends have been identified in consumer research, where Carù and Cova cautioned against the "ideological view [across disciplines of experiential research] that tends to consider every experience as extra-ordinary" [25, p. 268] - perspectives that recall the "search for intense pleasures and high arousal [rather than] the tepid mediocrity of everyday life" [25, p. 278] in Romantic ideals [55] whose relevance to the conditions of contemporary life is increasingly tenuous [134].

\section{ORDINARY EXPERIENCE}

Human life consists largely of ordinary experiences: going to work (or working from home), shopping for groceries, playing with a pet, and so on. When asked about recent life experiences, ordinary experiences are those described with a perfunctory gloss (e.g., "it's ok, I guess"; "nothing much happened"; "she's really getting into the scratching post") - or omitted from the narrative entirely [3, 133]. Extra-ordinary experiences come to mind more readily - being promoted, winning the lottery, or the death of a pet are unlikely to occur frequently. (If these experiences did occur regularly, that in itself would be extra-ordinary.)

Somewhat paradoxically, academic accounts say little about the experiential qualities of ordinary experiences, reflecting a prevailing tendency to understate or eschew consideration of ordinary experiences in the wider literature. Ordinary experiences, when mentioned at all, are typically deployed in comparisons that favour the extra-ordinary. Abrahams conceptualises extra-ordinary experiences as "more intense, framed and stylized practices" [3, p. 50], whereas ordinary experiences correspond to everyday life and the routine. Similarly, Arnould and Price [9] describe extraordinary experience in terms of spontaneity, and the absence of rigid expectations; again, ordinary experience is linked to mere routine. Duerden et al. acknowledge the value of studying ordinary experiences, but only through recourse to the extra-ordinary - "so they [ordinary experiences] do not detract from desired extraordinary experiences" [43, p. 201] - characterising ordinary experiences as those that require conscious attention without producing strong emotions.

Some works are less biased, however, and investigate both ordinary and extra-ordinary experiences with equal seriousness. Bhattacharjee et al. conceptualised ordinary experiences as "common, frequent, and within the realm of everyday life ... independent of any inferiority or superiority" [13, p. 2], and over a series of studies observed their increasing benefit to happiness with age. More specifically, their work suggests that the benefits of ordinary experiences increase with age due to a declining need for self-definition through memorable (i.e., extra-ordinary) experiences. Similarly, Irvin argues for the aesthetic appeal of ordinary experiences - defined as simple, everyday experiences, lacking closure, and characterised by fragmented awareness - which "animate our day-to-day existence" [60, p. 40], and represent a source of considerable satisfaction. Schmitt goes further, suggesting that "mundane experiences of medium intensity may in fact be the precondition for happiness. As such, they have an important role to play in enriching our ordinary, daily lives" [134, p. 251-252].

\subsection{Ordinary Experience in HCI}

A variety of $\mathrm{HCI}$ research has engaged with concepts related to ordinary experience. Some scholars aim to minimise the attentional burdens of interaction: Pohl and Murray-Smith describe a continuum of focused-casual interactions [119] that vary in terms of effort and attention; implicit [135] and peripheral interaction [10] research pertains to interfaces that support unaware (e.g., gazebased) or unintentional technology engagement (e.g., with public displays); others yet investigate non-intrusive and non-disruptive "subtle" interaction [118] as a desirable quality of technology use. Notably, these accounts remain largely silent on the experience of engaging with such technologies.

In contrast, works on "unremarkable" $[109,148]$ or "mundane" interactions [e.g., 40] emphasise the routine character of engagement, examining typically commonplace technologies that are no longer novel, having been fully integrated into daily life - they have become ordinary. Crucially, this view conceptualises "ordinariness [as] something that we $d o$; rather than simply being a stable feature of the world, it is actively managed and achieved in the course of interaction ... produced and recognised by the parties to an interaction ... [and] relative to particular communities and activities; it is a feature of forms of competent language use for groups of language users" [38, p. 24, emphasis added]. In short, ordinariness is collectively produced by literate beings in interaction [also see 133].

User Experience (UX) research has recently started investigating the notion of ordinary experience at work, where users often interact with automated or semi-automated processes. For Meneweger et al. [96], the (extra-)ordinary is best represented on a continuum, where ordinariness obtains in experience "that someone has no specific memory of, attributes no specific value to, or requires hardly any of someone's attention" [96, p. 219; emphasis added]. Ordinary experiences may be recalled as a gloss, but their specific qualities are typically inaccessible. In contrast, extra-ordinary [or "unordinary", 96] experiences are memorable, valued, or demand attention. From these authors' perspective, the (extra-)ordinariness of experience is continually (re)determined in the present, and these temporal dynamics are of primary relevance, rather than categories of ordinary or extra-ordinary experience as such.

What is meant by "attributing no specific value" to an experience, however, is less clear. Unfortunately, the paper itself [96] is somewhat ambivalent as to the specific meaning of 'value'. We may, however, speculate that unvalued experiences are particular to contexts where engagement is typically involuntary (e.g., workplaces), which suggests limited relevance to videogame play.

According to Clemmensen and colleagues, ordinary experiences "have no specific value, are hardly memorable, do not attract attention, and happen when users interact directly [or] indirectly with a system" [30, p. 6-7; emphasis added]. Nevertheless, they consider such experiences a key characteristic of UX at work, requiring further research. Findings from their two-week study of greenhouse workers' practices indicate that ordinariness is produced only by literate (or expert) users in interaction, substantiating prior conceptual work [38]. Their study design, which employed an ad-hoc 


\begin{tabular}{lcl}
\hline Element & Relevant Literature & Description \\
\hline Familiar & {$[3,6,22,30,38,41,63,72,79,137,151]$} & Games' salient features become familiar as literacy develops through play \\
Moderate emotion & {$[22,43,50,112,137,141]$} & Diffuse emotional responses to the developing scene of play \\
Co-attentive & {$[7,24,35,70,96,112,127,137,138,141,147]$} & A capacity for awareness as balanced across the videogame and outside world \\
Abstractly memorable & {$[84,96,100,147,148]$} & $\begin{array}{l}\text { A general or abstract recollection of the play situation, game plot, and other } \\
\text { structural features may be retained }\end{array}$ \\
\hline
\end{tabular}

Table 1: Elements of ordinary PX.

UX instrument and the AttrakDiff, is worth further notice for operationalising ordinary UX as "middle-of-the-scale" - a temporally stable pattern of moderately positive ratings (or neutral ratings, on the AttrakDiff's semantic differential). However, none of the scales employed by Clemmensen et al. immediately suggest themselves as a suitable measure of ordinary UX - indeed, whereas the authors interpret their qualitative data as further evidence for measuring ordinary experience as "middle-of-the-scale", some statements [e.g., "Repulsive or pleasing? No. It is a work tool"; 30, p. 18] may instead suggest the limited relevance of AttrakDiff items to the system under study.

Speculating on the subsequent directions of ordinary experience research, Meneweger et al. [96] are optimistic regarding its fruitful adaptation to other HCI research contexts, including leisure. Acknowledging that each approach described in this section has seen success within their specific context, none seem individually capable of characterising ordinary experiences of videogame play. It is for this reason that we now turn to consider the yet-unexamined qualities of ordinary PX.

\section{ORDINARY PX}

Players derive pleasure from ordinary experiences of videogame play $[112,146]$ - yet accounting for these engagements proves difficult (e.g., "it's more about progressing rather than enjoying the game [laughs] which sounds stupid but ..." [146, p. 73]). The vocabularies of videogames and player experience are so steeped in the extra-ordinary that we have no words to describe alternative ways that play can be desirable [also see 5].

Existing accounts of ordinary experience are not readily applicable to player experience: many approaches primarily concern utilitarian contexts [e.g., the workplace; 30, 96], or efficient task navigation [e.g., finding a parking lot near the dentist; 131] - circumstances that arguably bear little resemblance to common notions of play as a voluntary leisure activity $[35,160]$. Consequently, these accounts mainly consider ordinary experience only at the site of its rupture into the extra-ordinary [e.g., getting lost on the way to the dentist; 131]. Beyond a common interest in everyday routines, approaches aiming to minimise user attention towards interaction [e.g., 10,135] also seem misaligned with desirable experiences of play (a point we discuss in further detail below). Similarly, conceptualisations of ordinary experience in consumer research typically consider it only in terms of "common" and "frequent" experience $[13,97]$ to differentiate it from "uncommon" and "infrequent" extraordinary experiences. They say little about the experiential qualities of ordinariness.

In the following, we hence outline an account of ordinary experience as it pertains to player-computer interaction (see Table 1 for a summary). Qualities of ordinary PX were derived from these works on UX, HCI, and consumer research, as well as game studies.
We illustrate these qualities by means of several game examples, based on our personal experiences and those described in games scholarship. Before we articulate the qualities of ordinary PX, however, some preliminary points should be raised. First, ordinary PX is local to the specific personal context of videogame play. For example, the extra-ordinary experience of completing a boss fight with limited health remaining is incommensurable with that of winning the lottery; similarly, Katamari Forever [47] is likely to produce more ordinary PX for those who have played prior games in the series. Hence, the game examples and personal experiences detailed below may not reflect other players' views of ordinary PX. Second, we emphasise that ordinary PX does not merely reflect boredom. While the former might be seen as boring to others, the continued existence of game elements likely to evoke ordinary PX (e.g., grinding levels in a role-playing game) demonstrates their desirability. Indeed, boredom can be extra-ordinary, and such experiences are more clearly unpleasant; for example, when a game's final boss is much easier than expected, or when sitting through spectacular but ultimately lengthy animations [e.g., as in Final Fantasy VII; see 27]. Third, our conceptualisation situates the importance of ordinary experiences in their frequency and regularity, which help organise and structure life events into a coherent whole. To elaborate: whereas some authors cast extra-ordinary experiences as "highly emotional, meaningful, unique, and having the power to transform[, representing] an escape from common everyday life" [43, p. 201], we argue that the opposite is also true: the ordinary is itself an escape; a relief from the intense extra-ordinary experiences that wear out, and wear on, the self [12]. If extra-ordinary experiences are essential as moments of self-definition, meaning, and intense emotion, ordinary experiences represent the circumstances in which life can continue at all.

\subsection{Familiar}

The familiarity of ordinary PX refers to what "goes without saying”, the tacit knowledge, muscle memory, or general competences (i.e., literacies) developed through previous experience in similar circumstances [3, 30, 38, 96]. Extra-ordinary PX is characterised by novelty, which may result from steadily increasing challenge [i.e., as in flow; 33], plot twists [17], and so on.

Familiarity with videogame play develops primarily through direct interaction - with "hands wrapped around input devices, eyes on screens, and ears directed at speakers" [72, p. 16], players develop embodied literacies through more precise motor control, and greater recognition of visual, aural, and haptic game elements [41, 63, 72, 151]. Familiarity also pertains to the ideas, events, and tropes that games convey through representation [6, 64, 79, 151]; indeed, a game's intended audience may primarily consist of players who can interpret these representational qualities in ways that correspond to particular cultural literacies $[80,150]$. Put another way: through 
habit, players incorporate aspects of the play situation into bodily knowledge that can be accessed more readily [i.e., habituation; $75,122]$, and these literacies (e.g., WASD controls) can generalise to perceptibly similar circumstances.

Some game designs evoke familiarity through the convergence of interaction and representation: in depicting broadly accurate trainadjacent locales, and providing a custom interface resembling actual train controls, the Densha de Go! series [e.g., 145] also signifies an intent to represent the actually regulated behaviours imposed on train drivers in the outside world. Other games become familiar through a serial aesthetic of design that "folds difference into the repetition [of game elements] and so creates a sense of iterative progression" [143], contributing both structure and variation to play. Diablo III: Reaper of Souls [15], for example, deploys a serial aesthetic via recurring enemy abilities, item modifiers, and area tilemaps, which are collectively rearticulated in greater rifts and regular seasonal resets. To be familiar with Reaper of Souls is to recognise and respond to these salient features [64] during play - to identify the posthumous danger posed by enemies with the Vortex and Fire Enchanted attributes, feel the changes in crusader play produced by seasonal item variations, or know the procedurallygenerated patterns that determine where area exits could be located.

Players and game-external circumstances can also influence familiarity by deviating from normative behaviours [22] - holding a controller upside-down, wearing a blindfold, or playing a local multiplayer game in a public library will likely increase the novelty of the play situation. Familiarity, and hence ordinary PX, is multiply determined in direct interaction, representation, and the introduction of atypical factors into the play situation.

\subsection{Moderate Emotion}

Moderate emotion reflects more diffuse or gentle emotional responses to play. In other words, the emotional qualities of ordinary PX are 'moderate' only in that they are not excessively strong - neither intensely positive or negative, nor highly arousing or relaxing $[43,121]$. Note that this is not an absence of experiential qualities [cf. 30] - while these emotions are not strongly felt, their absence would instead suggest anhedonia. Extra-ordinary PX, in contrast, is often marked by a pronounced sense of enjoyment $[94,96]$, or intense emotional responses [e.g., 16].

Much of Xenoblade Chronicles 2 [98], for example, provokes moderate emotion: navigating its environments, gathering resources, completing quests, and watching cutscenes is generally pleasant, and somewhat stimulating, but never intense. Unusual monster names such as 'Implacable Dylan' might prompt a smile, and melodrama in the game's narrative is even occasionally affecting Xenoblade is by no means dull, or lacking in personality - however, the moderate emotions elicited by the game contribute to its appeal.

Considering technology use in general, Meneweger et al. argue that experiential qualities such as emotion may become less prominent over time, in that "users may pay initial attention to trust as a component of the experience, whereas after several trustful interactions, it might become less prominent in the users' experience" [96, p. 226]. This would reflect emotional habituation - and if similar interactions and experiences were found in other games, they might generalise to those games as well. Indeed, habituation and generalisation effects have been observed to attenuate emotional arousal over repeated play sessions with first-person shooter games [50] rather than seeking more gratuitous stimuli in response, however, long-time players recognise that play "can become a routine activity among other everyday routine activities", and begin to "find pleasure in more or less monotonous gaming experiences" [112, p. 232]. Game developers' conceptualisation of 'cozy' experiences, which obtain in "low-stress environment[s] ... where players have a lower state of arousal" [137, p. 1], also speaks to the desirability of moderate emotion in PX.

\subsection{Co-Attentive}

The co-attentiveness of ordinary PX refers to a balance of player attention across events in the videogame and the outside world $[30,70,96]$. In this sense, the objects of player attention are unremarkable; "perceptually available yet practically invisible in use" [148, p. 402, emphasis added]. However, contrary to notions of peripheral [10], intersecting [109], or implicit interaction [68, 135], co-attentiveness is "not equivalent to not noticing" [148, p. 402] - rather, it emphasises attention that is shared across the game and the outside world. For example, the second author often plays roguelike games on their (muted) laptop or handheld console while friends or family members watch and discuss a movie together nearby. Attending and contributing to these conversations remains possible without interrupting play. Extra-ordinary PX, conversely, involves distraction from the outside world: the player's full attention is consumed by the videogame, limiting awareness of external events (e.g., a burning smell from the oven).

Co-attentive play may be social $[7,141,147]$, involve separate media engagements [24, 112, 127], or other events [e.g., waiting for the correct bus stop; 35, 70]. Indeed, these examples illustrate that co-attentive play can be seen as a response to a wider trend towards technology designs and media formations that permeate daily life [106]: similar interaction styles are facilitated by other screen media [e.g., TV, livestreaming; 5, 115, 161] and emerging technologies [e.g., smart devices; 111]. In their work on screen ecologies, Carter et al. trace player accounts of EVE Online [26] that produce 'boring' styles of play, whose low attentional requirements produce "low registers of engagement" [24, p. 41], similar to the "everyday background activity" [138, p. 503] of idle game play. The co-attentiveness of ordinary PX does not, however, only describe periodic engagement - online games such as Guild Wars 2 [8], for example, have been observed to facilitate low-intensity experiences of engagement that support casual sociality [137].

Some game designs intentionally make co-attentive play central to the experience. The Longing [142], for instance, incorporates idle game mechanics [see also 138] to deliberate effect, as the playercharacter's actions are subject to protracted real-time delays that stretch over minutes, and even hours. In the meantime, players may attend to other activities - house chores, other games, or work while waiting for the 'pinging' sound that indicates task completion. Alternatively, the player-character can be instructed to wander randomly through the labyrinthine game environment, potentially discovering an unexplored area. The Longing also invites players to simply watch the player-character as they traverse the game world, or hack away at crystals - acts of mindful contemplation that may be experienced as quite extra-ordinary (as the second author of this work observed). 


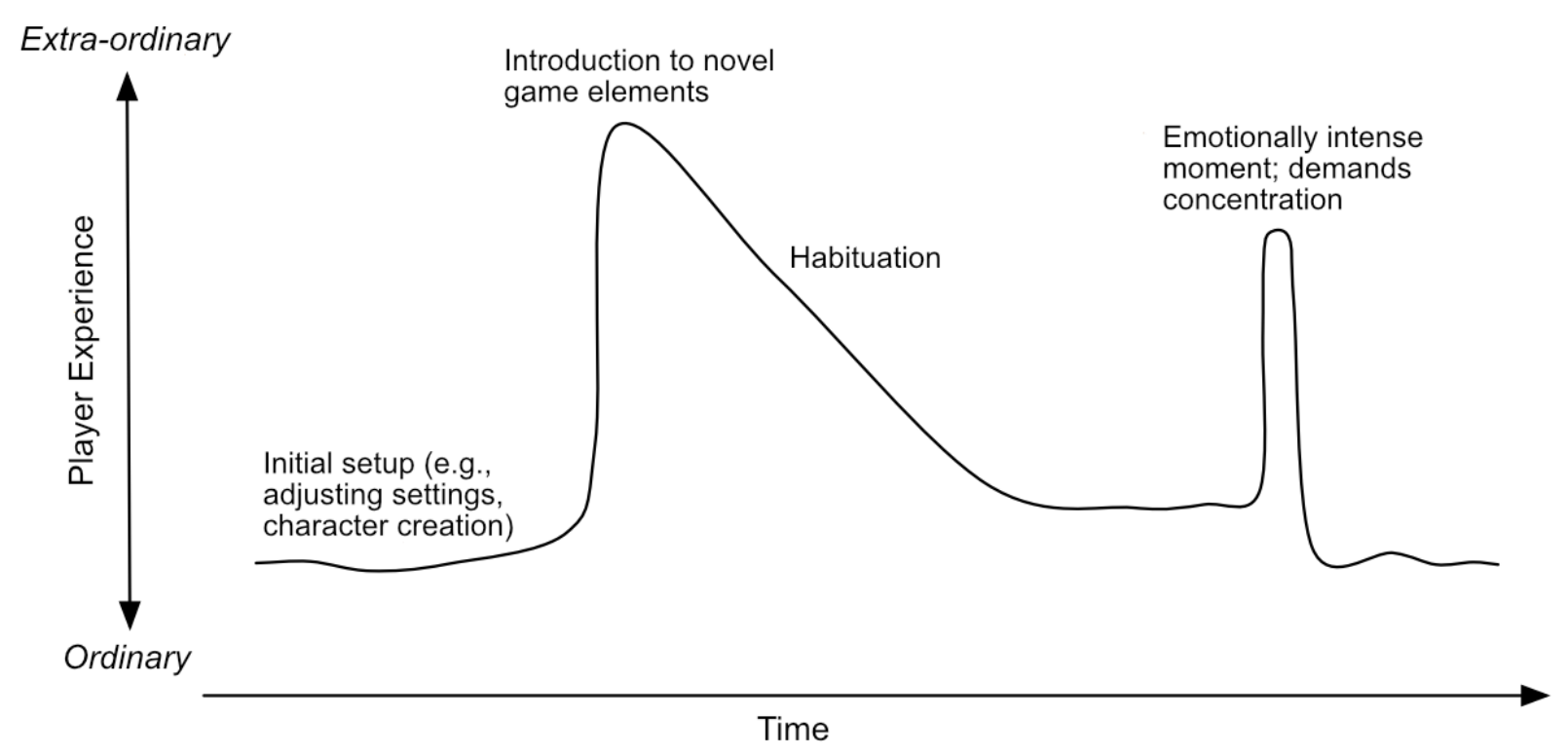

Figure 1: The temporal dynamics of (extra-)ordinary PX, represented as a continuum. Adapted from Meneweger et al. [96].

In emphasising movement, navigation, and environmental appreciation, walking simulators [or 'walkers'; 103] often invite coattentive play. In Proteus [76], for example, interactions are limited to movement and perception, and while the gameworld is alive and compelling, its navigation makes few demands on players' cognitive resources. Two exceptions that prove the rule are WORLD4 [101], which demands focused attention to four near-identical viewports during navigation [102], and Slave of God [88], whose pulsating nightclub environment demands attention by overwhelming the senses [73]. The aesthetics of aural and visual excess that both games deploy marks them as unusual within their genre, and emphasises the influence of focused attention for extra-ordinary PX.

\subsection{Abstractly Memorable}

Ordinary PX is abstractly memorable in that recollections are recognisably incomplete. That is, rather than remembering a specific experience episode, the abstract memory of ordinary PX is a gloss; a "vague gestalt impression" [100] of activity and emotion across the physical and virtual locales of play. These memories are available for conscious recollection [cf. implicit memory; 49], but details are perceptibly absent. Extra-ordinary PX produces more vivid memories of the play situation; upon their recollection, players may find themselves reliving (to a lesser degree) the emotional and physical responses originally experienced.

Final Fantasy VII's [140] tower defence mini-game at Fort Condor arguably lends itself to abstract memory; its relevance to the game's plot, themes, and ensemble cast is somewhat tenuous, premised only on opposing the forces of global capital in their attempts to steal a giant egg. When forming a defence, players can select from a reasonably varied pool of unit types, but combatants on both sides move and fight at an almost comically slow pace. While clear, episodic memories of Fort Condor are possible, their formation is likely more dependent on other aspects of the play situation - explaining the scene to a spectating family member, for example, or deploying only a single unit type. Separately, in eschewing conventional modes of temporality and narrative form, and embracing the surreal, Lake of Roaches [99] comes to resemble a series of impressions - fishing trip, hotel, the lake, a Roach King - whose recollection seems necessarily incomplete. Memories of playing Lake of Roaches are more likely to reflect players' overall experience with the game; a summary judgement lacking further detail.

The prevalence of peak experience studies and retrospective selfreport data in HCI games literature represent obstacles to finding evidence of PX that is unmemorable, or memorable only in the abstract. Self-report data are of some use, however, in demonstrating a relationship between specific memories and extra-ordinary PX. A study of memorable Pokémon GO experiences [84] indicates, through its coding scheme, that memorable PX emerged in part from the game's novelty - in being a 'newbie', doing things in the game for the 'first time', the 'hype' around the game in the 'early days' of its release, and (in what the authors term 'hysteria') the sometimes extra-ordinary lengths to which players went to catch rare pokémon. Moreover, memorable PX was also seen to provoke a variety of strong emotional responses, including thrill, frustration, and embarrassment [84]. Ethnographic research provides more direct evidence that ordinary $\mathrm{PX}$ is characterised in part by an absence of specific memories. In one study [147], participants who watched a previously recorded play session noted their failure to remember the event in detail. For these participants, the experience of 'not remembering' was foregrounded in "a seeming disjuncture between their [participants'] memories of gaming, and the recordings" [147, p. 137], whereby participants reported surprise and discomfort at their distraction from play or the outside world, their apparent passivity, and few visible signs of enjoyment.

\subsection{The (Extra-)Ordinary PX Continuum}

Ordinary and extra-ordinary PX reflect complementary approaches within HCI games research. Their characterisation as opposite ends of a continuum, as suggested by Meneweger et al. [96], is productive in illustrating how they relate (see Figure 1). Movement between 
the poles of ordinary and extra-ordinary PX occurs in interaction - as rapidly or slowly as the player's own response to the play situation over time. PX can quickly become more extra-ordinary, for example, if a player is surprised by an unfamiliar enemy whose defeat demands their full attention. Using the same example, a similarly abrupt shift towards ordinary PX may occur if the player immediately returns to a more peaceful locale afterwards (e.g., to recover health). Slower movements towards the ordinary may reflect habituation to increasingly familiar subject matter [96]; a slow progression into extra-ordinary PX may emerge from the dawning realisation that a well-practiced boss fight might finally be won. Players with more substantially relevant literacies may also intentionally work to make their experiences more extra-ordinary; for example, by playing a horror game with friends in the dark [see 112], or finding new ways to interact with other players [22].

Relations between ordinary PX elements - familiarity, moderate affect, abstract memory, and co-attentiveness - are well-evidenced in cognitive psychology: habituation, the process through which play becomes familiar, catalyses the capacities for co-attentive and emotionally moderate experiences [122]. As players become more literate in a particular game - or with elements that obtain across many game designs [e.g., 78, 110] - play becomes increasingly unlikely to elicit intense emotions [22, 84, 112], or distract players from other everyday activities such as cooking pasta, or watching television [24, 63, 112].

A substantial body of research has found that experiences of strong emotion are more often remembered in detail [e.g., see 69]. Crucially, focused attention towards the salient causes of intense emotion contributes to their memorisation and later retrieval [69], and preliminary work has found support for these relationships in the videogame context [65], albeit only for short-term recall.

Each dimension is to some degree necessary and complementary in producing ordinary PX. To summarise: ordinary PX originates in familiarity towards the videogame, which players enact via literacies. Familiarity attenuates both the cognitive demand of play (facilitating co-attentiveness), and emotional responses to stimuli that are no longer novel. In the absence of strong emotion and focused attention towards play, memory blurs; recollections of the scene are recognisably incomplete.

\section{DISCUSSION}

Beyond its status as a domain of games research in its own right, understanding player experience is important for a range of $\mathrm{HCI}$ games scholarship, including applied games, gamified systems, design, and wellbeing. Almost the entirety of research to date has investigated extra-ordinary experiences; treating games as "dramatic machines" [156] uniquely suited to provide optimal or intensely emotional experiences. Although some scholars have indicated that more ordinary experiences with videogames can be desirable [e.g., 138], ordinary PX is yet to receive serious attention in HCI games research. The present work contributes to PX theory development by articulating a conceptualisation of ordinary PX that accounts for the desirable aspects of day-to-day engagement with videogames. While we draw from works on ordinary UX [and its distinction from extra-ordinary experience; 30,96$]$, our conceptualisation extends these rather vague notions in specifying the familiar, emotionally moderate, co-attentive, and abstractly memorable nature of ordinary PX. In approaching ordinary and extra-ordinary PX as poles of a continuum between which PX varies over time, the present work contributes an extended perspective on player experience that complements existing knowledge of extra-ordinary PX.

In the following, we discuss our conceptualisation of ordinary PX with respect to its implications for empirical research, understanding what constitutes player experience, and relations between games and wellbeing.

\subsection{Implications for PX}

"The first issue prohibiting good evaluation of entertainment technologies is the inability to define what makes a system successful" [90, p. 142].

As we have shown, PX research to date has focused on extraordinary experiences, implying that good PX - or PX worth investigating - corresponds to these experiences alone. Understanding how extra-ordinary PX became prominent, and why HCI games research has pursued broadly-applicable frameworks of good or "most important" [104, p. 4] PX elements, helps contextualise the conceptual importance of ordinary PX.

Despite popular appeal, videogames have historically struggled to establish cultural legitimacy [31, 80]. Games research, by association, has undergone a similar struggle [2]: in academia, for example, the CHI Games and Play subcommittee has only existed since 2016, and "might be a bit of an outsider" [117, p. 8]. HCI games research, and PX research in particular, has primarily claimed legitimacy as a distinct area of study through associations with UX [e.g., 104] and psychology [149]. Drawing from these areas has imparted PX research with theoretical, conceptual, and methodological substance; foundations that have undoubtedly contributed to the recognition and development of games and play research at CHI. However, these fundamental claims to relevance increasingly resemble a 'legitimacy trap' [39] that narrows the field of possibilities for further growth [also see 11]. In presenting ordinary PX, we have in part attempted to illustrate the substantive limits of current PX research.

Alongside work that develops existing PX research concepts in greater detail [e.g., 32, 35, 81, 116], more varied ways of theorising player experience [e.g., 139] are increasingly necessary to understand changes in game design. MOBA games, for example, have thwarted existing PX evaluation methods because they "...lack most of the commonly occurring positive components of PX" [66, p. 2270]. Research into other design trends over the past decade mobile games [84, 93], idle games [34, 138], Twine games [46, 53], and walking simulators $[102,103]$ - also indicates that a greater breadth of PX concepts is needed. As our examples of familiar, emotionally moderate, co-attentive, and abstractly memorable experiences have shown, ordinary PX can help explain the appeal of many of these games in ways that existing PX concepts may not.

In suggesting that ordinary PX can help explain these games' appeal, we also highlight the variety of experiences that videogames are increasingly designed to support. We consequently hope to displace notions that general frameworks of 'optimal' or 'good' PX can be defined at all [cf. 86]. It is clear that "games and game development keep changing, [creating] new and specific situations" [42, p. 518] that games scholarship must address - but these changes in game-making practice fundamentally resist attempts to "find a 
good combination of measures that can identify a valuable player experience holistically, efficiently, and cheaply" [42, p. 519]. Returning to the (reinterpreted) quote that opened this section [90], the issue at hand is the inability to define success for all systems.

Another implication pertains to areas of research aiming to "optimise" PX with respect to flow or novelty [e.g., 129, 130]; for example, to predict churn in mobile games [129]. These approaches overlook the often ordinary experiences that accompany mobile play [particularly with live service games; 4], overestimating the predictive utility of positive extra-ordinary PX in models of player behaviour. Developing models that account for the varied patterns of engagement with live service games may be particularly important, as their designs tend to reward routine engagement across a number of time periods. For example, Destiny 2 [21] incorporates daily, weekly, and 'seasonal' objectives that justify repeated play. For Destiny 2 and its contemporaries, ordinary PX is embedded in the designed routines intended to structure engagement [148].

5.1.1 Implications for Extra-Ordinary PX. Our conceptualisation also suggests new ways of thinking about extra-ordinary PX in particular, as a means to describe trajectories of PX over time [96]. During crucial early periods of engagement (i.e., onboarding), for example, the (extra-)ordinary PX continuum could help demonstrate changes in the intended experience, alongside other temporally-sensitive approaches [114].

Naturally, there are many in-game events that players would prefer to experience as being extra-ordinary; scenes that designers intend to be remarkable rather than mundane. For example, if pentakills (i.e., killing all five members of the opposing team in succession) became a more ordinary experience in League of Legends [126] tournaments, it might suggest serious issues with game balance, and result in a less desirable spectator experience. Games that exploit a combination of ordinary and extra-ordinary PX demonstrate their complementary benefits: the plot twists of Bioshock [1] and Braid [108], for example, depend on familiar experiences; the extra-ordinary PX these games evoked could only occur because following directions and rescuing princesses were considered ordinary.

\subsection{Ordinary PX and Wellbeing}

Ordinary PX provides another account regarding the ways that games can improve wellbeing. Wellbeing benefits associated with videogame play have been repeatedly claimed in $\mathrm{HCI}$ games literature [e.g., 59, 151, 153]. Common explanations for this relationship come from psychology (e.g., self-determination theory, mood management theory), including extra-ordinary experience literature [e.g., flow; 105, 120]. The ways that HCI games research approaches wellbeing, then, is bound up in many of the same ideas regarding peak experience that we have sought to complicate here. As before, we do not suggest that these perspectives are wrong - but in ordinary PX we find an explanation independent of videogames' distraction potential [59] or need-satisfying qualities [151], and an alternative account of play's social benefits [153]. In presenting an alternative way of thinking about games and wellbeing, we identify another limit of established approaches to PX research.

Videogames' capacity to distract players from issues in the outside world [i.e., as part of emotion-based coping; 59] has featured heavily in research linking games and wellbeing [e.g., 67, 152]. Our conceptualisation of ordinary PX, however, has drawn attention to work demonstrating that more literate players often engage co-attentively with games typically theorised as immersive or flowinducing [e.g., 24, 112, 137]. Collectively, these instances challenge prevalent assumptions about how play can benefit player wellbeing - for example, the capacity to talk through the events of a bad day while playing competitive multiplayer games does not seem to follow from experiencing flow or immersion [154], but rather co-attentiveness. Considering co-attentiveness in this context is not only to observe its prevalence outside mobile and casual game play [24, 74]; rather, it is also to see other ways to derive wellbeing benefits from engagement with more conventional videogames.

During play, elements of ordinary PX provide a respite from unpleasant feelings experienced in daily life $[124,155]$. Games suited to coping behaviours are often familiar to their players [137], and support emotional moderation [or regulation; 51] - the attenuation of negative emotions through their transferral into 'bodily knowledge' that is felt less keenly. Over time, ordinary experiences of play structure and reproduce life; they represent habitual behaviour through which a degree of stability can be attained [122]. In this sense, ordinary PX supports wellbeing through the maintenance of routine, contributing to a pleasant sense of self-abeyance [12]: a temporary suspension of, and relief from, being in relation with the world. From this perspective, the regularity of wellbeing is more fundamental than its intensity [36]. This shift corresponds to a change in measurement. For example, using self-report scales anchored from 'not at all' to 'extremely' implies that wellbeing obtains in uncommon, intensely positive experiences. Future scholarship on games and wellbeing may alternatively benefit from measures anchored in terms of frequency [e.g., 37].

It is no revelation that older adults regularly play videogames, and for a variety of reasons [19, 20,48]. Consumer research has indicated that relations between ordinary experience and wellbeing increase with age [13] due to changes in self-definition practices. More specifically, "as people get older, their focus may shift from discovering who they are through ... unique endeavours like ziplining through the rainforest to living who they are by spending time in their preferred ways" [13, p. 10]. However, it remains unclear whether similar relationships manifest with respect to videogame play: Gamers (particularly men) may self-identify as such at younger ages $[80,82,136]$, but this identity is arguably substantiated at least partly through the habitual, unremarkable, and ordinary play experiences that coalesce into the literacies recognised as legitimate within the culture [71, 150]. Investigating the ways that age and identity formation strategies intersect with ordinary PX - and to what extent these relationships differ from those identified in consumer research - would therefore represent an interesting vector for future research.

Another avenue for investigation relates to potential design implications for games and game-adjacent artefacts that promote wellbeing [e.g., 29, 153], as these domains are likely to benefit from more varied approaches to design [28]. Specifically, designing for wellbeing through ordinary PX could emphasise the comfort of repetition and familiarity, and emotionally gentle situations [e.g., as in 'cozy' games; 137]. 


\subsection{Limitations and Open Questions}

The present work has adapted work from a variety of research domains to propose a detailed conceptualisation of ordinary player experience. However, we emphasise that this paper does not represent the final word on the topic; indeed, we invite scholarship that develops or challenges our approach to ordinary PX. Crucially, beyond the supporting literature presented, our conceptualisation awaits direct empirical validation in the context of videogame play.

Our synthesis of varied theoretical views does complicate the $o p$ erationalisation of familiarity, moderate emotion, co-attentiveness, and abstract memory for use in HCI games research. Indeed, questions of measurement have emerged in prior ordinary experience research: Meneweger et al., for example, highlight that UX research provides "no explicit reflections on how to access [ordinary] experiences" [96, p. 225], and suggest ethnographic and practice-led [87] methods. These approaches are demonstrably effective [e.g., $3,148]$. UX research has suggested quantitative approaches to operationalising ordinary experience as mid-scale ratings [30], yet this risks conflating ordinary with mediocre experience. As such, substantial challenges in measuring the properties of ordinary PX remain. Considering familiarity in terms of direct interaction, representational qualities, and contextual factors reflects a broader issue of defining and measuring expertise [62]. Moderate emotion is more straightforward, given existing self-report measures, but skewed distributions present their own challenges in analysis [159]. Unfortunately, the novelty of biometric measurement may itself facilitate more extra-ordinary PX [but see 50]. Self-report or behavioural measures of task demand [e.g., see 18] may implicitly assess co-attentiveness - alternatively, observational methods are more complex and time-consuming to implement, but may provide a more direct assessment [e.g., 45]. Finally, while the nebulous nature of abstract memory appears to stymie current approaches to measurement, distinguishing between specific memories of play and the broader play session (e.g., in survey prompts) may prove effective.

Although the present work has focused entirely on videogames, boardgame research [128] indicates that similar forms of ordinary PX may obtain in tabletop contexts. More specifically, the ways that players collaboratively distribute and maintain knowledge of the game state, while retaining sociality when circumstances permit [128] speaks to co-attentiveness, particularly among more familiar players. The potential nuances in ordinary boardgame PX, relative to our own conceptualisation, would represent a valuable site of further inquiry.

Gameful and applied games scholarship may also benefit from considering ordinary PX, in light of prior research in non-leisure settings $[30,96]$. In these contexts, the utility of ordinary PX seems particularly contingent on design intent: applied game designs, for example, may intend to develop tacit knowledge (i.e., becoming familiar, and ordinary) or confront players with more discordant (i.e., extra-ordinary) scenarios. However, ordinary PX seems more consistently relevant to gamified systems, as these designs often aim to support the initiation and maintenance of routine behaviours [28] Gamifying workplace environments presents practical and ethical issues, however, in that the potential for voluntary engagement is inherently foreclosed by their "hierarchical and unbalanced power relations" [157, p. 174], which would appear to negate any benefits of gameful intervention.

Finally, while our conceptualisation was specifically developed with ordinary player experience in mind, it may also facilitate more granular investigations of ordinary user experience with technology. This application is particularly salient, having drawn from the "abstract experiencing" of ordinary interaction described by Meneweger et al. [96] when constructing the co-attentive and abstractly memorable elements of ordinary PX. Whereas prior ordinary UX research has focused on the workplace [30, 96], our conceptualisation may lend itself to investigating ordinary experiences of leisurely technology use (e.g., habitual smartphone interactions $[89,111]$; watching and producing video content online $[115,161]$; devices intended to afford "subtle" interaction [118]). Having articulated elements of ordinary PX, our conceptualisation represents a response to calls for further engagement with the "different nuances" [30, 96, p. 7; p. 227] of ordinary user experience.

\section{CONCLUSION}

HCI games research has historically treated videogames as "dramatic machines" [156] uniquely suited to support extra-ordinary experiences of flow, pronounced enjoyment, immersion, or intense emotion. While memorable, such experiences are rare and do not reflect more prevalent, ordinary experiences of play. In this paper, we have argued for the importance of such experiences: synthesising works in HCI, consumer research, and game studies, the present work has presented a conceptualisation of ordinary PX, as distinct from extra-ordinary experience. Ordinary PX is characterised by familiar, emotionally moderate, co-attentive, and abstractly memorable experiences with videogames. We have, moreover, described ordinary and extra-ordinary PX as poles of a continuum on which PX varies over time. As such, the present work contributes an extension to player experience theory that both challenges and complements existing work on extra-ordinary PX in HCI games research.

\section{ACKNOWLEDGMENTS}

Special thanks to Dylan Schneider for early discussions that helped confirm the relevance and direction of this work.

\section{REFERENCES}

[1] $2 \mathrm{~K}$ Boston and 2K Australia. 2007. Bioshock. Videogame [PC]. 2K Games, California, USA.

[2] Espen Aarseth. 2001. Computer Game Studies, Year One. Game Studies 1, 1 (2001).

[3] Roger D. Abrahams. 1986. Ordinary and Extraordinary Experience. In The Anthropology of Experience. University of Illinois Press, 45-72.

[4] Kati Alha, Jani Kinnunen, Elina Koskinen, and Janne Paavilainen. 2018. Free-toPlay Games: Paying Players' Perspective. In Proceedings of the 22nd International Academic Mindtrek Conference. ACM.

[5] Ien Ang. 1982/1985. Dallas and the Ideology of Mass Culture. In Watching Dallas. 86-116. Della Couling (Trans.). Routledge, London, England.

[6] Thomas Apperley. 2010. Digital Game Ecologies. In Gaming Rhythms: Play and Counterplay from the Situated to the Global. Institute of Network Cultures, Amsterdam, Netherlands, 11-33.

[7] Thomas Apperley. 2010. The Social Milieu. In Gaming Rhythms: Play and Counterplay from the Situated to the Global. Institute of Network Cultures, Amsterdam, Netherlands, 67-83.

[8] ArenaNet. 2012. Guild Wars 2. Videogame [PC]. NCSoft, Seongnam, Republic of Korea. 
[9] Eric J. Arnould and Linda L. Price. 1993. River Magic: Extraordinary Experience and the Extended Service Encounter. Journal of Consumer Research 20, 1 (1993), 24-45.

[10] Saskia Bakker, Elise van den Hoven, and Berry Eggen. 2015. Peripheral Interaction: Characteristics and Considerations. Personal and Ubiquitous Computing 19, 1 (2015), 239-254.

[11] Lauren Berlant. 2011. Cruel Optimism. Duke University Press, North Carolina, USA.

[12] Lauren Berlant. 2011. Slow Death (Obesity, Sovereignty, Lateral Agency). In Cruel Optimism. Duke University Press, Durham, USA, 95-120.

[13] Amit Bhattacharjee and Cassie Mogilner. 2014. Happiness from Ordinary and Extraordinary Experiences. Fournal of Consumer Research 41, 1 (2014), 1-17. https://doi.org/10.1086/674724

[14] Max V. Birk, Ioanna Iacovides, Daniel Johnson, and Regan L. Mandryk. 2015 The False Dichotomy Between Positive and Negative Affect in Game Play. In Proceedings of the 2015 Annual Symposium on Computer-Human Interaction in Play. ACM, 799-804.

[15] Blizzard Entertainment. 2014. Diablo III: Reaper of Souls. Videogame [PC] Blizzard Entertainment, California, USA

[16] Julia Ayumi Bopp, Elisa D. Mekler, and Klaus Opwis. 2016. Negative Emotion, Positive Experience?: Emotionally Moving Moments in Digital Games. In Proceedings of the 2016 CHI Conference on Human Factors in Computing Systems. ACM, 2996-3006.

[17] Julia Ayumi Bopp, Klaus Opwis, and Elisa D. Mekler. 2018. "An Odd Kind of Pleasure": Differentiating Emotional Challenge in Digital Games. In Proceedings of the 2018 CHI Conference on Human Factors in Computing Systems. ACM.

[18] Nicholas D. Bowman and Ron Tamborini. 2012. Task Demand and Mood Repair: The Intervention Potential of Computer Games. New Media \& Society 14, 8 (2012), 1339-1357.

[19] Jeffrey E. Brand, Jan Jervis, Patrice M. Huggins, and Tyler W. Wilson. 2019. Digital Australia 2020. Report. IGEA.

[20] Jeffrey E. Brand, Jan Jervis, Patrice M. Huggins, and Tyler W. Wilson. 2019. Digital New Zealand 2020. Report. IGEA.

[21] Bungie. 2017. Destiny 2. Videogame [PC]. Activision, California, USA.

[22] Marcus Carter. 2015. The First Week of the Zombie Apocalypse: The Influences of Game Temporality. Journal of Gaming \& Virtual Worlds 7, 1 (2015), 59-75.

[23] Marcus Carter, John Downs, Bjorn Nansen, Mitchell Harrop, and Martin Gibbs. 2014. Paradigms of Games Research in HCI: A Review of 10 Years of Research at CHI. In Proceedings of the First ACM SIGCHI Annual Symposium on Computer Human Interaction in Play. ACM, 27-36.

[24] Marcus Carter, Bjorn Nansen, and Martin Gibbs. 2014. Screen Ecologies, MultiGaming and Designing for Different Registers of Engagement. In Proceedings of the First ACM SIGCHI Annual Symposium on Computer-Human Interaction in Play. ACM, 37-46.

[25] Antonella Carù and Bernard Cova. 2003. Revisiting Consumption Experience: $\mathrm{A}$ More Humble but Complete View of the Concept. Marketing Theory 3, 2 (2003) 267-286.

[26] CCP Games. 2003. EVE Online. Videogame [PC]. CCP Games, Reykjavík, Iceland.

[27] ChairForceOne. 2013. Final Fantasy VII 7: Knights of the Round Summon HD Video. Retrieved 12 September 2020 from https://www.youtube.com/watch?v= sv9aI5RkoeQ

[28] Vanessa Wan Sze Cheng, Tracey Davenport, Daniel Johnson, Kellie Vella, and Ian B. Hickie. 2019. Gamification in Apps and Technologies for Improving Mental Health and Well-Being: Systematic Review. FMIR Mental Health 6, 6 (2019).

[29] Vanessa Wan Sze Cheng, Tracey Davenport, Daniel Johnson, Kellie Vella, Jo Mitchell, and Ian B. Hickie. 2020. Naturalistic Evaluation of a Sport-Themed Mental Health and Wellbeing App Aimed at Men (MindMax) that Incorporates Applied Video Games and Gamification. Internet Interventions 20 (2020).

[30] Torkil Clemmensen, Morten Hertzum, and Jose Abdelnour-Nocera. 2020. Ordinary User Experiences at Work: A Study of Greenhouse Growers. ACM Transactions on Computer-Human Interaction 27, 3 (2020).

[31] Mia Consalvo and Christopher A. Paul. 2013. Welcome to the Discourse of the Real: Constituting the Boundaries of Games and Players. In Proceedings of the 8th International Conference on the Foundations of Digital Games.

[32] Anna Cox, Paul Cairns, Pari Shah, and Michael Carroll. 2012. Not Doing but Thinking: The Role of Challenge in the Gaming Experience. In Proceedings of the SIGCHI Conference on Human Factors in Computing Systems. ACM, 79-88.

[33] Mihaly Csikszentmihalyi and Judith LeFevre. 1989. Optimal Experience in Work and Leisure. Journal of Personality and Social Psychology 56, 5 (1989), 815-822.

[34] Joe Cutting, David Gundry, and Paul Cairns. 2019. Busy Doing Nothing? What do Players do in Idle Games? International fournal of Human-Computer Studies 122 (2019), 133-144.

[35] Sebastian Deterding. 2016. Contextual Autonomy Support in Video Game Play: A Grounded Theory. In Proceedings of the 34th Annual ACM Conference on Human Factors in Computing Systems. ACM, 3931-3943.
[36] Ed Diener, Ed Sandvik, and William Pavot. 2009. Happiness is the Frequency, Not the Intensity, of Positive Versus Negative Affect. In Assessing Well-Being: The Collected Works of Ed Diener, Ed Diener (Ed.). Springer, 213-231.

[37] Ed Diener, Derrick Wirtz, William Tov, Chu Kim-Prieto, Dong-won Choi, Shigehiro Oishi, and Robert Biswas-Diener. 2010. New Well-being Measures: Short Scales to Assess Flourishing and Positive and Negative Feelings. Social Indicators Research 97, 2 (2010), 143-156.

[38] Paul Dourish. 2004. What we Talk About When we Talk About Context. Personal and Ubiquitous Computing 8 (2004), 19-30.

[39] Paul Dourish. 2019. User Experience as Legitimacy Trap. Interactions 26, 6 (2019), 46-49.

[40] Paul Dourish, Connor Graham, Dave Randall, and Mark Rouncefield. 2010. Theme Issue on Social Interaction and Mundane Technologies. Personal and Ubiquitous Computing 14, 1 (2010), 171-180.

[41] Jon Dovey and Helen W. Kennedy. 2006. Networks of Technicity. In Game Cultures: Computer Games as New Media. Open University Press, Berkshire, England, 63-83.

[42] Anders Drachen, Pejman Mirza-Babaei, and Lennart E. Nacke. 2018. Frontlines in Games User Research. In Games User Research, Anders Drachen, Pejman Mirza-Babaei, and Lennart E. Nacke (Eds.). Oxford University Press, Oxford, England, 509-519.

[43] Mat D. Duerden, Neil R. Lundberg, Peter Ward, Stacy T. Taniguchi, Brian Hill, Mark A. Widmer, and Ramon Zabriskie. 2018. From Ordinary to Extraordinary: A Framework of Experience Types. Journal of Leisure Research 49, 3-5 (2018), 196-216.

[44] Entertainment Software Association. 2019. 2019 Essential Facts About the Computer and Video Game Industry. Report.

[45] James E. Folkestad, Brian McKernan, Stephanie Train, Rosa Mikeal Martey, Matthew G. Rhodes, Kate Kenski, Adrienne Shaw, Jennifer Stromer-Galley, Benjamin A. Clegg, and Tomek Strzalkowski. 2018. The Temporal Attentive Observation (TAO) Scale: Development of an Instrument to Assess Attentive Behavior Sequences During Serious Gameplay. Technology, Knowledge, and Learning 23 (2018), 65-81.

[46] Jane Friedhoff. 2013. Untangling Twine: A Platform Study. In Proceedings of DiGRA 2013.

[47] Genki. 2009. Katamari Forever. Videogame [PlayStation 3]. Bandai Namco Games, Nakano, Japan.

[48] Kathrin M. Gerling. 2015. Long-Term Use of Motion-Based Video Games in Care Home Settings. In Proceedings of the CHI Conference on Human Factors in Computing Systems. ACM.

[49] Peter Graf and Daniel L. Schacter. 1985. Implicit and Explicit Memory for New Associations in Normal and Amnesiac Subjects. Fournal of Experimental Psychology: Learning, Memory, and Cognition 11, 3 (1985), 501-518.

[50] Matthew Grizzard, Ron Tamborini, John L. Sherry, René Weber, Sujay Prabhu, Lindsay Hahn, and Patrick Idzik. 2015. The Thrill Is Gone, but You Might Not Know: Habituation and Generalisation of Biophysiological and Self-Reported Arousal Responses to Video Games. Communication Monographs 82, 1 (2015), 64-87.

[51] James J. Gross. 2015. Emotion Regulation: Current Status and Future Prospects. Psychological Inquiry 26, 1 (2015), 1-26.

[52] Carl Gutwin, Christianne Rooke, Andy Cockburn, Regan L. Mandryk, and Benjamin Lafreniere. 2016. Peak-End Effects on Player Experience in Casual Games. In Proceedings of the 2016 CHI Conference on Human Factors in Computing Systems. 5608-5619.

[53] Alison Harvey. 2014. Twine's Revolution: Democratisation, Depoliticisation, and the Queering of Game Design. GAME - Games as Art, Media, Entertainment 3 (2014), 95-107.

[54] Hit-Point. 2014. Neko Atsume: Kitty Collector. Videogame [Android]. Nagoya, Japan.

[55] Morris B. Holbrook. 1997. Romanticism, Introspection, and the Roots of Experiential Consumption: Morris the Epicurean. Consumption, Markets and Culture 1, 2 (1997), 97-163.

[56] Morris B. Holbrook, Robert W. Chestnut, Terence A. Oliva, and Eric A. Greenleaf. 1984. Play as a Consumption Experience: The Roles of Emotions, Performance, and Personality in the Enjoyment of Games. Fournal of Consumer Research 11 (1984), 728-739.

[57] Morris B. Holbrook and Elizabeth C. Hirschman. 1982. The Experiential Aspects of Consumption: Consumer Fantasies, Feelings, and Fun. fournal of Consumer Research 9, 2 (1982), 132-140.

[58] Ioanna Iacovides and Anna L. Cox. 2015. Moving Beyond Fun: Evaluating Serious Experience in Digital Games. In Proceedings of the 33rd Annual ACM Conference on Human Factors in Computing Systems. ACM, 2245-2254.

[59] Ioanna Iacovides and Elisa D. Mekler. 2019. The Role of Gaming During Difficult Life Experiences. In Proceedings of the 2019 CHI Conference on Human Factors in Computing Systems. ACM.

[60] Sherri Irvin. 2008. The Pervasiveness of the Aesthetic in Ordinary Experience. The British Journal of Aesthetics 48, 1 (2008), 29-44. 
[61] Glena H. Iten, Sharon T. Steinemann, and Klaus Opwis. 2018. Choosing to Help Monsters: A Mixed-Method Examination of Meaningful Choices in NarrativeRich Games and Interactive Narratives. In Proceedings of the 2018 CHI Conference on Human Factors in Computing Systems. ACM.

[62] Darshana Jayemanne. 2017. Performativity in Art, Literature, and Videogames. Springer International, Cham, Switzerland.

[63] Darshana Jayemanne. 2017. Physical Wit: Games and the 'Tactile Unconscious'. In Performativity in Art, Literature, and Videogames. Springer International, Cham, Switzerland, 189-226.

[64] Darshana Jayemanne. 2017. Serial Aesthetics - Gaming's Metamorphic Bodies and Baudelaire's 'Argot Plastique'. In Performativity in Art, Literature, and Videogames. Springer International, Cham, Switzerland, 161-188.

[65] Eui Jun Jeong and Frank A. Biocca. 2012. Are There Optimal Levels of Arousal to Memory? Effects of Arousal, Centrality, and Familiarity on Brand Memory in Video Games. Computers in Human Behavior 28 (2012), 285-291.

[66] Daniel Johnson, Lennart Nacke, and Peta Wyeth. 2015. All about that Base: Differing Player Experiences in Video Game Genres and the Unique Case of MOBA Games. In Proceedings of 33rd Annual ACM Conference on Human Factors in Computing Systems. 2265-2274.

[67] Daniel Johnson, Peta Wyeth, Penelope Sweetser, and John Gardner. 2012. Personality, Genre and Videogame Play Experience. In Proceedings of the 4th International Conference on Fun and Games. ACM, 117-120.

[68] Wendy Ju and Larry Leifer. 2008. The Design of Implicit Interactions: Making Interactive Systems Less Obnoxious. Design Issues 24, 3 (2008), 72-84.

[69] Elizabeth A. Kensinger. 2009. Remembering the Details: Effects of Emotion Emotion Review 1, 2 (2009), 99-113.

[70] Brendan Keogh. 2014. Paying Attention to Angry Birds: Rearticulating Hybrid Worlds and Embodied Play through Casual iPhone Games. In The Routledge Companion to Mobile Media, Gerard Goggin and Larissa Hjorth (Eds.). Routledge Ltd, 267-275.

[71] Brendan Keogh. 2018. From Hackers to Cyborgs. In A Play of Bodies: How We Perceive Videogames. MIT, Cambridge, USA, 167-192.

[72] Brendan Keogh. 2018. A Play of Bodies: How We Perceive Videogames. MIT Cambridge, USA

[73] Brendan Keogh. 2018. To Feel Sights and Sounds. In A Play of Bodies: How We Perceive Videogames. MIT, Cambridge, USA, 109-136.

[74] Brendan Keogh. 2018. Touching the Looking Glass. In A Play of Bodies: How We Perceive Videogames. MIT, Cambridge, USA, 51-74.

[75] Brendan Keogh. 2018. With Thumbs in Mind. In A Play of Bodies: How We Perceive Videogames. MIT, Cambridge, USA, 75-108.

[76] Ed Key and David Kanaga. 2013. Proteus. Videogame [PC]. Twisted Tree.

[77] Rilla Khaled. 2018. Questions Over Answers: Reflective Game Design. In Playful Disruption of Digital Media, Daniel Cermak-Sassenrath (Ed.). Springer, Singapore, 3-27.

[78] Daniel King, Paul Delfabbro, and Mark Griffiths. 2010. Video Game Structural Characteristics: A New Psychological Taxonomy. International fournal of Mental Health and Addiction 8, 1 (2010), 90-106.

[79] Geoff King and Tanya Krzywinska. 2006. Gameplay and its Contexts. In Tomb Raiders and Space Invaders: Videogame Forms and Contexts. Palgrave Macmillan, London, England, 8-75.

[80] Graeme Kirkpatrick. 2013. The Formation of Gaming Culture. In Computer Games and the Social Imaginary. Polity Press, 70-97.

[81] Madison Klarkowski, Daniel Johnson, Peta Wyeth, Cody Phillips, and Simon Smith. 2018. Don't Sweat the Small Stuff: The Effect of Challenge-Skill Manipulation on Electrodermal Activity. In Proceedings of the 2018 Annual Symposium on Computer-Human Interaction in Play. ACM, 231-242.

[82] Carly A. Kocurek. 2015. Coin-Operated Americans - Rebooting Boyhood at the Video Game Arcade. University of Minnesota Press, Minneapolis, USA.

[83] Korea Creative Content Agency. 2018. 2018 White Paper on Korean Games Summary. Report. Korea Creative Content Agency (KOCCA).

[84] Elina Koskinen, Dale Leorke, Kati Alha, and Janne Paavilainen. 2019. Player Experiences in Location-Based Games: Memorable Moments with Pokémon GO. In Augmented Reality Games I, Vladimir Geroimenko (Ed.). Springer, Switzerland, 95-116.

[85] Annakaisa Kultima. 2009. Casual Game Design Values. In Proceedings of the 13th International MindTrek Conference. ACM, 58-65.

[86] Annakaisa Kultima and Jaakko Stenros. 2010. Designing Games for Everyone The Expanded Game Experience Model. In Proceedings of the International Academic Conference on the Future of Game Design and Technology. ACM, 6673.

[87] Kari Kuutti and Liam J. Bannon. 2014. The Turn to Practice in HCI: Towards a Research Agenda. In Proceedings of the CHI Conference on Human Factors in Computing Systems. ACM, 3543-3552.

[88] Stephen Lavelle. 2012. Slave of God. Videogame [PC]

[89] Kai Lukoff, Cissy Yu, Julie Kientz, and Alexis Hiniker. 2018. What Makes Smartphone Use Meaningful or Meaningless? Proceedings of the ACM on Interactive, Mobile, Wearable and Ubiquitous Technologies 2, 1 (2018), 1-26.
[90] Regan L. Mandryk, Kori M. Inkpen, and Thomas W. Calvert. 2006. Using Psychophysiological Techniques to Measure User Experience with Entertainment Technologies. Behaviour \& Information Technology 25, 2 (2006), 141-158.

[91] Tim Marsh and Brigid Costello. 2012. Experience in Serious Games: Between Positive and Serious Experience. In International Conference on Serious Games Development and Applications. Springer, 255-267.

[92] Abraham H. Maslow. 1962. Toward a Psychology of Being. Simon and Schuster

[93] Christian McCrea. 2017. Pokémon's Progressive Revelation: Notes on 20 Years of Game Design. Mobile Media \& Communication 5, 1 (2017), 42-46.

[94] Elisa D. Mekler, Julia Ayumi Bopp, Alexandre N. Tuch, and Klaus Opwis. 2014 A Systematic Review of Quantitative Studies on the Enjoyment of Digital Entertainment Games. In Proceedings of the 32nd Annual ACM Conference on Human Factors in Computing Systems. ACM, 927-936.

[95] Elisa D. Mekler, Ioanna Iacovides, and Julia Ayumi Bopp. 2018. "A Game that Makes You Question...": Exploring the Role of Reflection for the Player Experience. In Proceedings of the 2018 Annual Symposium on Computer-Human Interaction in Play. ACM, 315-327.

[96] Thomas Meneweger, Daniela Wurhofer, Verena Fuchsberger, and Manfred Tscheligi. 2018. Factory Workers' Ordinary User Experiences: An Overlooked Perspective. Human Technology 14, 2 (2018), 209-232.

[97] Kate E Min, Peggy J Liu, and Soo Kim. 2018. Sharing Extraordinary Experiences Fosters Feelings of Closeness. Personality and Social Psychology Bulletin 44, 1 (2018), 107-121.

[98] Monolith Soft. 2017. Xenoblade Chronicles 2. Videogame [Nintendo Switch]. Nintendo, Kyoto, Japan.

[99] Stephen Murphy. 2013. Lake of Roaches. Videogame [PC]

[100] Stephen Murphy. 2020. Betsy's Hospital GameFAQ. Retrieved 26 August, 2020 from http://harmonyzone.org/text/betsyhospital.html

101] Alexander Muscat. 2016. WORLD4. Videogame [PC]. Melbourne, Australia.

[102] Alexander Muscat and Jonathan Duckworth. 2018. WORLD4: Designing Ambiguity for First-Person Exploration Games. In Proceedings of the 2018 Annual Symposium on Computer-Human Interaction in Play. ACM, 341-351.

[103] Alexander Muscat, William Goddard, Jonathan Duckworth and Jussi Holopainen. 2016. First-Person Walkers: Understanding the Walker Experience through Four Design Themes. In Proceedings of the 1st International foint Conference of DiGRA and FDG.

[104] Lennart Nacke and Anders Drachen. 2011. Towards a Framework of Player Experience Research. In Proceedings of the Second International Workshop on Evaluating Player Experience in Games at FDG. ACM.

105] Jeanne Nakamura and Mihaly Csikszentmihalyi. 2002. The Concept of Flow. In Handbook of Positive Psychology, C. R. Snyder and Shane J. Lopez (Eds.). Oxford University Press, Oxford, England, 89-105.

[106] Angela Ndalianis. 2004. Neo-Baroque Aesthetics and Contemporary Entertainment. MIT Press, Cambridge, USA

[107] David Ng and Leslie June. 1985. Electronic Leisure and Youth: Kitchener Arcade Video Game Players. Society and Leisure 8, 2 (1985), 537-548.

[108] Number None. 2008. Braid. Videogame [Xbox 360]. Microsoft Game Studios, Redmond, USA.

[109] William Odom and Ron Wakkary. 2015. Intersecting with Unaware Objects. In Proceedings of the 2015 ACM SIGCHI Conference on Creativity and Cognition. 33-42.

[110] Mercè Oliva, Óliver Pérez-Latorre, and Reinald Besalú. 2018. 'Choose, Collect, Manage, Win!': Neoliberalism, Enterprising Culture and Risk Society in Video Game Covers. Convergence 24, 6 (2018), 607-622.

[111] Antti Oulasvirta, Tye Rattenbury, Lingyi Ma, and Eeva Raita. 2012. Habits Make Smartphone Use More Pervasive. Personal and Ubiquitous Computing 16 (2012), 105-114.

[112] Daniel Pargman and Peter Jakobsson. 2008. Do You Believe in Magic? Compute Games in Everyday Life. European fournal of Cultural Studies 11, 2 (2008), 225-243.

[113] Xiaolan Peng, Jin Huang, Alena Denisova, Hui Chen, Feng Tian, and Hongan Wang. 2020. A Palette of Deepened Emotions: Exploring Emotional Challenge in Virtual Reality Games. In Proceedings of the 2020 CHI Conference on Human Factors in Computing Systems. ACM.

[114] Falko Weigert Petersen, Line Ebdrup Thomsen, Pejman Mirza-Babaei, and Anders Drachen. 2017. Evaluating the Onboarding Phase of Free-to-Play Mobile Games: A Mixed-Method Approach. In Proceedings of the Annual Symposium on Computer-Human Interaction in Play. ACM, 377-388.

[115] Andrew M. Phelps and Mia Consalvo. 2020. Laboring Artists: Art Streaming on the Videogame Platform Twitch. In Proceedings of the 53rd Hawaii International Conference on System Sciences.

[116] Cody Phillips, Daniel Johnson, Madison Klarkowski, Melanie Jade White, and Leanne Hides. 2018. The Impact of Rewards and Trait Reward Responsiveness on Player Motivation. In Proceedings of the 2018 Annual Symposium on ComputerHuman Interaction in Play. ACM, 393-404.

117] Henning Pohl and Aske Mottelson. 2019. How we Guide, Write, and Cite at CHI In Extended Abstracts of the 2019 CHI Conference on Human Factors in Computing Systems. ACM. 
[118] Henning Pohl, Andreea Muresan, and Kasper Hornbæk. 2019. Charting Subtle Interaction in the HCI Literature. In Proceedings of the 2019 CHI Conference on Human Factors in Computing Systems. ACM, 1-15.

[119] Henning Pohl and Roderick Murray-Smith. 2013. Focused and Casual Interactions: Allowing Users to Vary their Level of Engagement. In Proceedings of the SIGCHI Conference on Human Factors in Computing Systems. ACM, 2223-2232.

[120] Gayle Privette. 1983. Peak Experience, Peak Performance, and Flow: A Comparative Analysis of Positive Human Experiences. Fournal of Personality and Social Psychology 45, 6 (1983), 1361-1368.

[121] Gayle Privette and Charles M. Bundrick. 1987. Measurement of Experience: Construct and Content Validity of the Experience Questionnaire. Perceptual and Motor Skills 65 (1987), 315-322.

[122] Felix Ravaisson. 1838/2008. Of Habit. Clare Carlisle and Mark Sinclair (Eds.) Continuum, London, England.

[123] Niklas Ravaja, Mikko Salminen, Jussi Holopainen, Timo Saari, Jari Laarni, and Aki Järvinen. 2004. Emotional Response Patterns and Sense of Presence During Video Games: Potential Criterion Variables for Game Design. In Proceedings of the Third Nordic Conference on Human-Computer Interaction. ACM, 339-347.

[124] Leonard Reinecke. 2009. Games and Recovery: The Use of Video and Computer Games to Recuperate from Stress and Strain. Fournal of Media Psychology 21, 3 (2009), 126-142.

[125] C. Scott Rigby and Richard M. Ryan. 2011. Dangerous Waters: The Addictive Undertow of Games. In Glued to Games: How Video Games Draw Us In and Hold Us Spellbound. Praeger, Santa Barbara, USA, 97-118.

[126] Riot Games. 2009. League of Legends. Videogame [PC]. Riot Games, California, USA.

[127] Melissa J. Rogerson and Martin Gibbs. 2018. Finding Time for Tabletop: Board Game Play and Parenting. Games and Culture 13, 3 (2018), 280-300.

[128] Melissa J. Rogerson, Martin R. Gibbs, and Wally Smith. 2018. Cooperating to Compete: the Mutuality of Cooperation and Competition in Boardgame Play. In Proceedings of the 2018 CHI Conference on Human Factors in Computing Systems ACM.

[129] Shaghayegh Roohi, Asko Relas, Jari Takatalo, Henri Heiskanen, and Perttu Hämäläinen. 2020. Predicting Game Difficulty and Churn Without Players. In Proceedings of the 2020 Annual Symposium on Computer-Human Interaction in Play. ACM.

[130] Shaghayegh Roohi, Jari Takatalo, Christian Guckelsberger, and Perttu Hämäläinen. 2018. Review of Intrinsic Motivation in Simulation-Based Game Testing. In Proceedings of the 2018 CHI Conference on Human Factors in Computing Systems. ACM, 1-13.

[131] J. Robert Rossman and Mathew D. Duerden. 2019. Designing Experiences Columbia University Press, New York, USA.

[132] Richard M. Ryan and Edward L. Deci. 2017. Motivation and Need Satisfaction in Video Games and Virtual Environments. In Self-Determination Theory: Basic Psychological Needs in Motivation, Development, and Wellness. Guilford, New York, NY, 508-531.

[133] Harvey Sacks. 1984. On Doing "Being Ordinary". In Structures of Social Action Studies in Conversation Analysis, J. Maxwell Atkinson and John Heritage (Eds.). Cambridge University Press, Cambridge, England, 413-429.

[134] Bernd H. Schmitt. 2000. Experiential Marketing: How to Get Customers to Sense, Feel, Think, Act, Relate. Simon and Schuster.

[135] Barış Serim and Giulio Jacucci. 2019. Explicating "Implicit Interaction": An Examination of the Concept and Challenges for Research. In Proceedings of the 2019 CHI Conference on Human Factors in Computing Systems. ACM, 1-16.

[136] Adrienne Shaw. 2012. Do You Identify as a Gamer? Gender, Race, Sexuality, and Gamer Identity. New Media \& Society 14, 1 (2012), 28-44.

[137] Tanya X. Short, Anthony Ordon, Dan Hurd, Chelsea Howe, Jake Forbes, Squirrel Eiserloh, Joshua Diaz, and Daniel Cook. 2017. Group Report: Coziness in Games: An Exploration of Safety, Softness, and Satisfied Needs. Report. projecthorseshoe. com/reports/featured/ph17r3.htm

[138] Katta Spiel, Sultan A. Alharthi, Andrew Jian-Lan Cen, Jessica Hammer, Lennart E Nacke, Z. O. Toups, and Tess Tanenbaum. 2019. "It Started as a Joke": On the Design of Idle Games. In Proceedings of the Annual Symposium on ComputerHuman Interaction in Play. ACM, 495-508.
[139] Katta Spiel and Kathrin Gerling. 2019. The Surrogate Body in Play. In Proceedings of the Annual Symposium on Computer-Human Interaction in Play. ACM, 397411.

[140] Square. 1997. Final Fantasy VII. Videogame [PlayStation]. Sony Computer Entertainment, California, USA.

[141] Reed Stevens, Tom Satwicz, and Laurie McCarthy. 2008. In-Game, In-Room, In-World: Reconnecting Video Game Play to the Rest of Kids' Lives. In The Ecology of Games: Connecting Youth, Games, and Learning, Katie Salen (Ed.). MIT Press, Cambridge, USA, 41-66.

[142] STUDIO SEUFZ. 2020. The Longing. Videogame [PC]. Application Systems Heidelberg, Heidelberg, Germany.

[143] David Surman. 2008. Notes on SuperFlat and its Expression in Videogames. Refractory: A fournal of Entertainment Media 13 (2008). https://web.archive. org/web/20200315182814/http://refractory.unimelb.edu.au/2008/05/23/noteson-superflat-and-its-expression-in-videogames-david-surman/

[144] Penelope Sweetser and Peta Wyeth. 2005. GameFlow: A Model for Evaluating Player Enjoyment in Games. Computers in Entertainment 3, 3 (2005).

[145] Taito. 2002. Densha de Go! 3 Tsūkin-hen. Videogame [PlayStation 2]. Taito, Tokyo, Japan.

[146] Helen Thornham. 2011. Articulating Pleasure: Gender, Technology, and Power. In Ethnographies of the Videogame: Gender, Narrative, and Praxis. Ashgate, Surrey, Enlgand, 47-76.

[147] Helen Thornham. 2011. Pleasure and the Imagined Gamer. In Ethnographies of the Videogame: Gender, Narrative, and Praxis. Ashgate, Surrey, England, 125-147.

[148] Peter Tolmie, James Pycock, Tim Diggins, Allan MacLean, and Alain Karsenty. 2002. Unremarkable Computing. In Proceedings of the CHI Conference on Human Factors in Computing Systems. ACM, 399-406.

[149] April Tyack and Elisa D. Mekler. 2020. Self-Determination Theory in HCI Games Research: Current Uses and Open Questions. In Proceedings of the $2020 \mathrm{CHI}$ Conference on Human Factors in Computing Systems. ACM.

[150] April Tyack and Peta Wyeth. 2017. Exploring Relatedness in Single-Player Video Game Play. In Proceedings of the 29th Australian Conference on Computer-Human Interaction. ACM, 422-427.

[151] April Tyack, Peta Wyeth, and Daniel Johnson, 2020. Restorative Play: Videogames Improve Player Wellbeing After a Need-Frustrating Event. In Proceedings of the 2020 CHI Conference on Human Factors in Computing Systems. ACM.

[152] Kellie Vella, Daniel Johnson, and Leanne Hides. 2015. Indicators of Wellbeing in Recreational Video Game Players. In Proceedings of the Annual Meeting of the Australian Special Interest Group for Computer Human Interaction. ACM, 613-617.

[153] Kellie Vella, Daniel Johnson, and Leanne Hides. 2015. Playing Alone, Playing With Others: Differences in Player Experience and Indicators of Wellbeing. In Proceedings of the 2015 Annual Symposium on Computer-Human Interaction in Play. ACM, 3-12.

[154] Kellie Vella, Daniel Johnson, and Jo Mitchell. 2016. Playing Support: Socia Connectedness Amongst Male Video Game Players. In Proceedings of the 2016 Annual Symposium on Computer-Human Interaction in Play Companion Extended Abstracts. ACM, 343-350.

[155] Greg Wadley. 2016. Mood-Enhancing Technology. In Proceedings of the 28th Australian Conference on Computer-Human Interaction. ACM, 326-332.

[156] Mark Weiser. 1994. Creating the Invisible Interface (Invited Talk). In Proceeding of the 7th Annual ACM Symposium on User Interface Software and Technology.

[157] Jennifer R. Whitson. 2013. Gaming the Quantified Self. Surveillance \& Society $11,1 / 2(2013), 163-176$

[158] Josef Wiemeyer, Lennart Nacke, Christiane Moser, and Florian 'Floyd' Mueller. 2016. Player Experience. In Serious Games: Foundations, Concepts, and Practice, Ralf Dörner, Stefan Göbel, Wolfgang Effelsberg, and Josef Wiemeyer (Eds.). Springer International Publishing, Switzerland, 243-272.

[159] Rand Wilcox. 2012. Introduction to Robust Estimation and Hypothesis Testing (3rd ed.). Elsevier, Amsterdam, Netherlands.

[160] Lisa A. Wing. 1995. Play is Not the Work of the Child: Young Children's Perceptions of Work and Play. Early Childhood Research Quarterly 10 (1995), 223-247.

[161] Donghee Yvette Wohn and Guo Freeman. 2020. Audience Management Practices of Live Streamers on Twitch. In ACM International Conference on Interactive Media Experiences. ACM. 